\title{
Structure analysis of $\mathrm{NiZr}_{2}$ in reciprocal and real space
}

\author{
N. Mattern ${ }^{1 *}$ J. Sakowski ${ }^{2}$, C. Baehtz ${ }^{3}$ \\ ${ }^{1}$ Leibniz-Institute for Solid State and Materials Research IFW Dresden \\ ${ }^{2}$ Department of Physics, University Rostock \\ ${ }^{3}$ Department for Material- and Geo -Sciences, Technical University Darmstadt \\ * Contact author; e-mail: n.mattern@ifw-dresden.de
}

Keywords: metallic glasses, high temperature X-ray diffraction, short-range order, thermal expansion, $\mathrm{Ni}_{33} \mathrm{Zr}_{67}$

\begin{abstract}
The temperature dependence of the geometric structure of amorphous and crystallized $\mathrm{Ni}_{33} \mathrm{Zr}_{67}$ was investigated by means of in situ synchrotron X-ray diffraction. The crystal structure parameters of the tetragonal $\mathrm{NiZr}_{2}$ phase were determined by the Rietveld refinement of the X-ray diffraction patterns. The lattice parameters $a_{0}, c_{0}$, and therefore the interatomic distances increase linearly with temperature. The behavior of the short-range order was analyzed by the corresponding atomic pair correlation functions $G(r)$. The position of the first maximum of $G(r)$ at $r_{1}$ decreases with temperature. The analysis of real space data of the crystallized state of $\mathrm{Ni}_{33} \mathrm{Zr}_{67}$ shows that the measured $G(r)$ function consists of several superimposed, non-resolved inter-atomic distances. The individual distances increase with temperature in agreement with the Rietveld analysis. The position of the first maximum $r_{1}$ is additionally influenced by the thermal broadening of the distance distributions leading to the "unphysical" reduction of $r_{1}$ with temperature.
\end{abstract}

\section{Introduction}

The short-range order in non-crystalline materials is revealed by the analysis of the atomic pair correlation function $G(r)$. For metallic glasses and metallic elemental liquids the position of the first maximum of $G(r)$ stays constant or even decreases with temperature [1,2]. The aim of this work was to compare the temperature behavior of the short-range order of a binary metallic glass with that of its corresponding crystalline state. $\mathrm{The} \mathrm{Ni}_{33} \mathrm{Zr}_{67}$ alloy was chosen because the amorphous state crystallizes isomorphous into $\mathrm{NiZr}_{2}$, and the alloy is related to the new class of Zr-based bulk metallic glasses [3].

\section{Experimental}

Amorphous $\mathrm{Ni}_{33} \mathrm{Zr}_{67}$ ribbons $3 \mathrm{~mm}$ in width and $25 \mu \mathrm{m}$ in thickness were prepared by means of rapid quenching technique from the melt. X-ray diffraction (XRD) patterns proofed the amorphous state of the as cast material. To analyze the temperature dependence of the crystal 
structure part of the ribbons was annealed under vacuum at $400^{\circ} \mathrm{C}$ for $30 \mathrm{~min}$. The annealed crystalline, and brittle ribbons were powderized and filled into a capillary. Temperature dependent X-ray diffraction patterns were recorded in Debye-Scherrer geometry at the powder diffraction beam-line B2 of HASYLAB. The experimental set-up $(\lambda=0.6993 \AA)$ consists of a STOE furnace and an image plate detector (OBI). The exposure time was $5-10 \mathrm{~min}$ per diagram depending on the DORIS current. To analyze the temperature behavior of the shortrange order, in situ XRD measurements were conducted at the high-energy beam-line BW5 of HASYLAB (wave length $\lambda=0.1076 \AA$ ). The experimental setup consists of a high temperature chamber and an image plate detector enabling to record diffraction pattern in transmission geometry within 30s and up to a scattering vector $q=200 \mathrm{~nm}^{-1}(q=4 \pi \sin \theta / \lambda)$ [4]. The amorphous as-quenched sample (stack of 20 ribbons) was stepwise measured and heated up to $400{ }^{\circ} \mathrm{C}$ where crystallization took place. Afterwards the sample was cooled down to $20{ }^{\circ} \mathrm{C}$ and the temperature dependent XRD measurement was repeated with the crystallized material.

\section{Results and discussion}

Figure 1 shows the XRD patterns of the crystallized $\mathrm{Ni}_{33} \mathrm{Zr}_{67}$ alloy measured from $800{ }^{\circ} \mathrm{C}$ to room temperature. The annealed material consists of the tetragonal $\mathrm{NiZr}_{2}$ phase. Up to the highest temperature $\mathrm{T}=800{ }^{\circ} \mathrm{C}$, tetragonal $\mathrm{NiZr}_{2}$ is registered only. The crystal structure

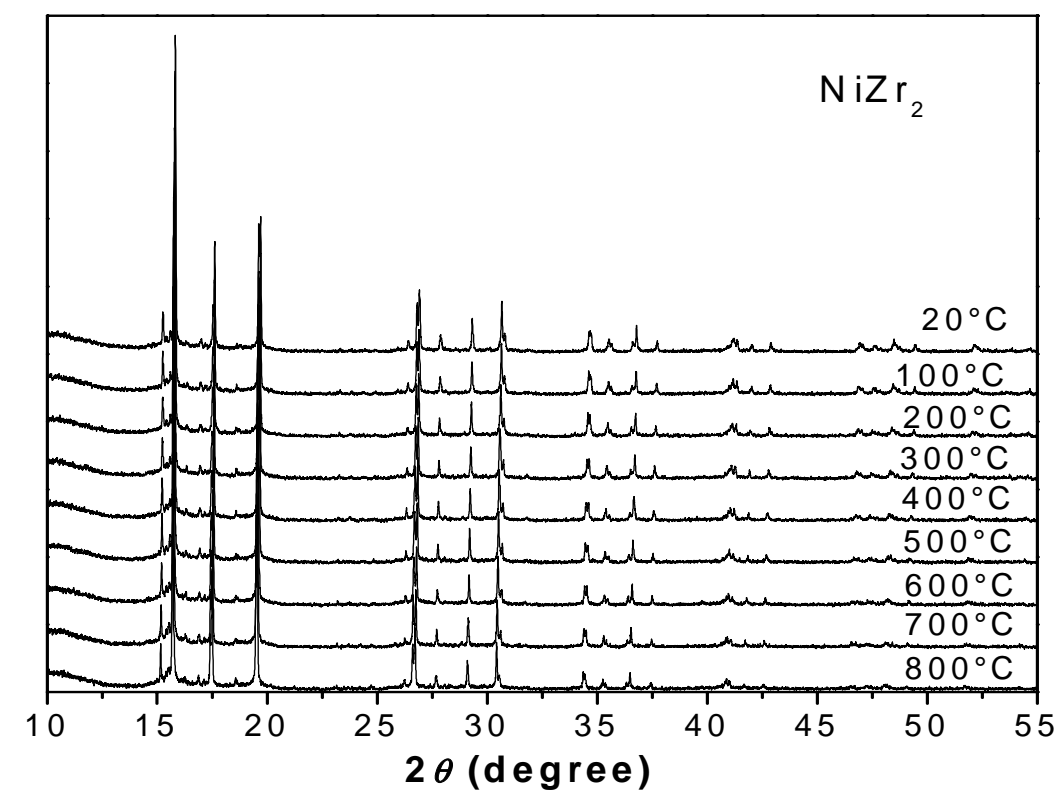

Figure 1. XRD patterns of crystallized NiZr 2 at elevated temperatures. 
$\mathrm{NiZr2}$

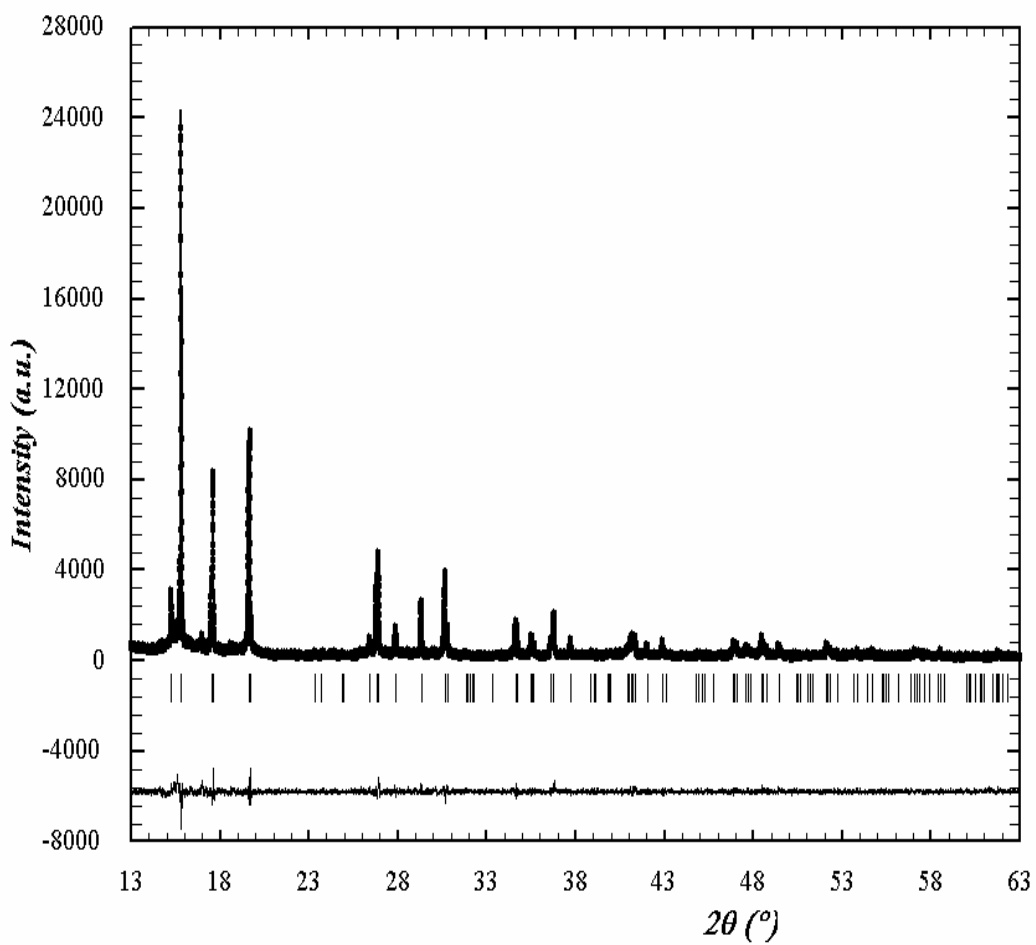

Figure 2. Rietveld plot of tetragonal $\mathrm{NiZr}_{2}$ at room temperature.

parameters were refined using FULLPROF software. Figure 2 shows as an example the Rietveld plot of the room temperature measurement. The calculated crystal structure parameters versus temperature are given in table 1. A linear thermal expansion is observed with the linear expansion coefficients $\alpha_{\mathrm{a}}=9.5 \times 10^{-6} / \mathrm{K}$, and $\alpha_{\mathrm{c}}=9.1 \times 10^{-6} / \mathrm{K}$ respectively. The position of the $\mathrm{Zr}$ atom at $8 h(x, x+1 / 2,0)$ stays constant. A linear increase of all inter-atomic distances with temperature follows from the crystal structure parameters given in table 2 .

Table 1. Crystal structure parameters of tetragonal NiZr 2 (S.G. 140).

\begin{tabular}{|l|c|c|c|c|c|c|c|c|}
\hline & $50^{\circ} \mathrm{C}$ & $100^{\circ} \mathrm{C}$ & $200^{\circ} \mathrm{C}$ & $300^{\circ} \mathrm{C}$ & $400^{\circ}$ & $500^{\circ} \mathrm{C}$ & $600^{\circ} \mathrm{C}$ & $800^{\circ} \mathrm{C}$ \\
\hline$a / \AA$ & 6.4905 & 6.4952 & 6.5030 & 6.5107 & 6.5186 & 6.5256 & 6.5328 & 6.5448 \\
\hline$c / \AA$ & 5.2679 & 5.2707 & 5.2752 & 5.2802 & 5.2859 & 5.2917 & 5.2987 & 5.3147 \\
\hline$x(8 h)$ & 0.8357 & 0.8360 & 0.8359 & 0.8356 & 0.8359 & 0.8359 & 0.8362 & 0.8359 \\
\hline$B / \AA^{-2}$ & 0.07 & 0.23 & 0.47 & 0.63 & 0.91 & 1.15 & 1.37 & 1.78 \\
\hline
\end{tabular}


The Rietveld method is based on the analysis of the positions and intensities of Bragg reflections. This allows us to determine the long-range average structure of the crystal. The shortrange order of any material can be characterized by its atomic pair correlation function, which is well known for amorphous substances [5]. The method of atomic pair correlation functions has recently been applied also to crystalline materials [6]. The reduced atomic pair correlation function $G(r)$ is obtained by the Fourier transform of the structure factor $S(q)$ [5]:

$$
G(r)=4 \pi \cdot r\left(\rho(r)-\rho_{0}\right)=\frac{2}{\pi} \cdot \int[S(q)-1] \cdot q \cdot \sin (q \cdot r) \cdot d q,
$$

where $\rho(r)$ is the atomic density pair correlation function, and $\rho_{0}$ is the mean atomic density. The structure factor $S(q)$ is calculated from the elastic scattered intensity in absolute units [5]. Large values of $q$ are required for resolution in $G(r)$ and to reduce the termination errors. Figure 3 shows the measured structure factors $S(q)$ of amorphous and crystallized $\mathrm{Ni}_{33} \mathrm{Zr}_{67}$ at room temperature. At elevated temperatures the heights of the maxima of $S(q)$ become reduced due to the temperature factor both for the amorphous and the crystallized sample. Figure 4 shows the corresponding atomic pair correlation functions $G(r)$. They differ clearly between the amorphous and the crystallized state due to the rearrangement of the atoms during the crystallization. The curves represent the superposition of the three partial atomic pair correlation function [5]. The two submaxima of the first maximum can be attributed to the nearest neighbor distances $\mathrm{Ni}-\mathrm{Zr}$, and $\mathrm{Zr}-\mathrm{Zr}$. The positions of the maxima are shifted and become broadened with temperature, which is related to the thermal expansion, and to the increase of the mean square displacement of the atoms.

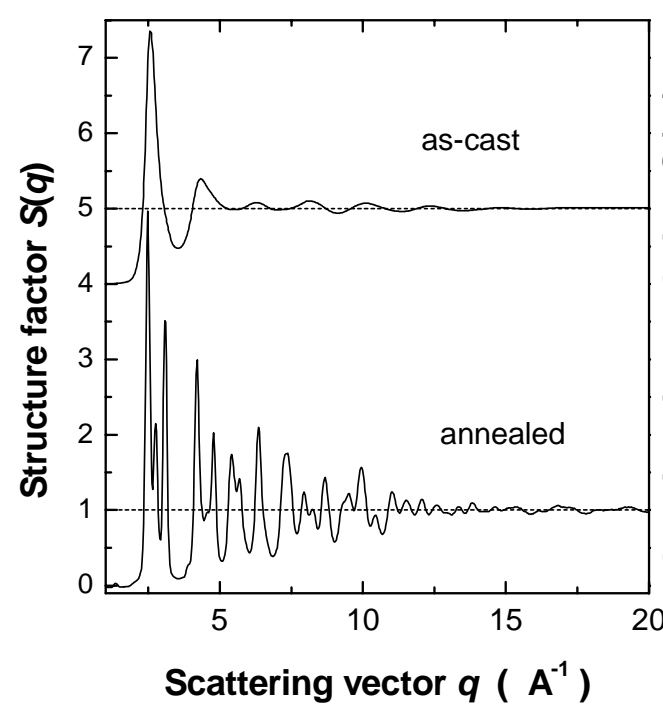

Figure 3. Structure factors $S(q)$ of amorphous and and crystallized $\mathrm{Ni}_{33} \mathrm{Zr}_{67}$.

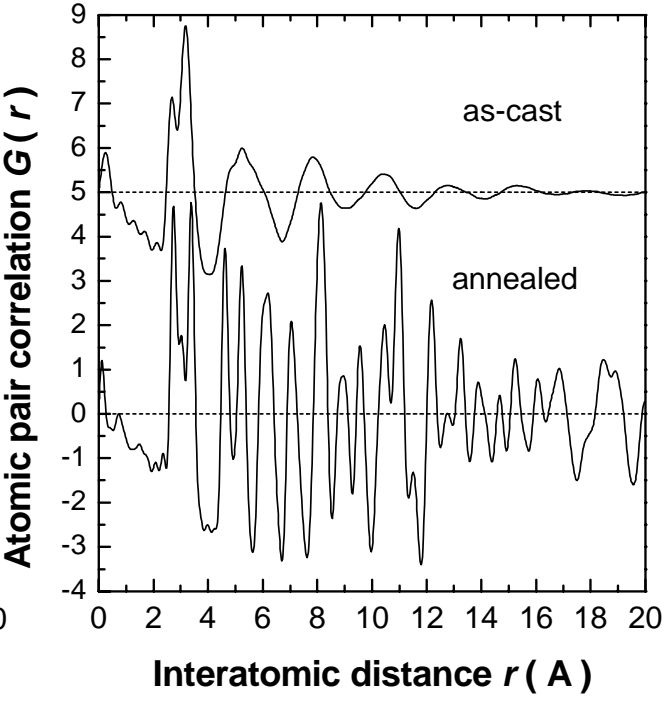

Figure 4. Atomic pair correlation functions of amorphous and crystallized $N_{33} Z r_{67}$. 


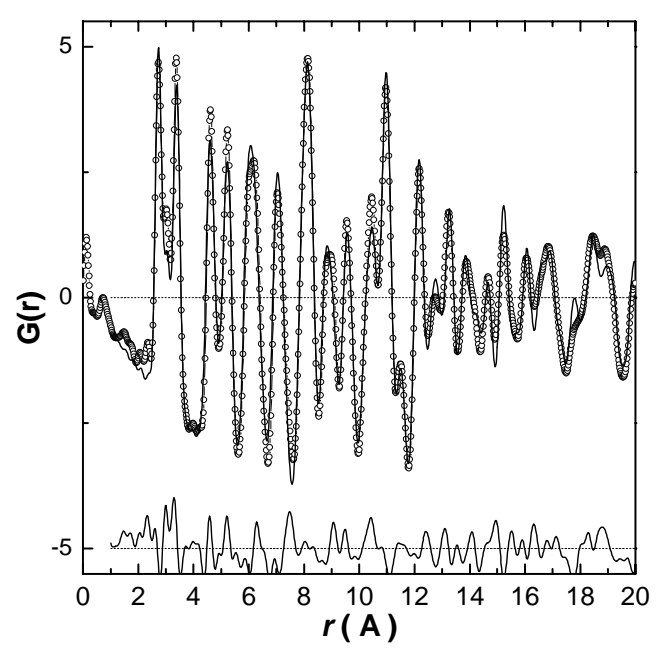

Figure 5. PDFFIT of crystallized NiZr.

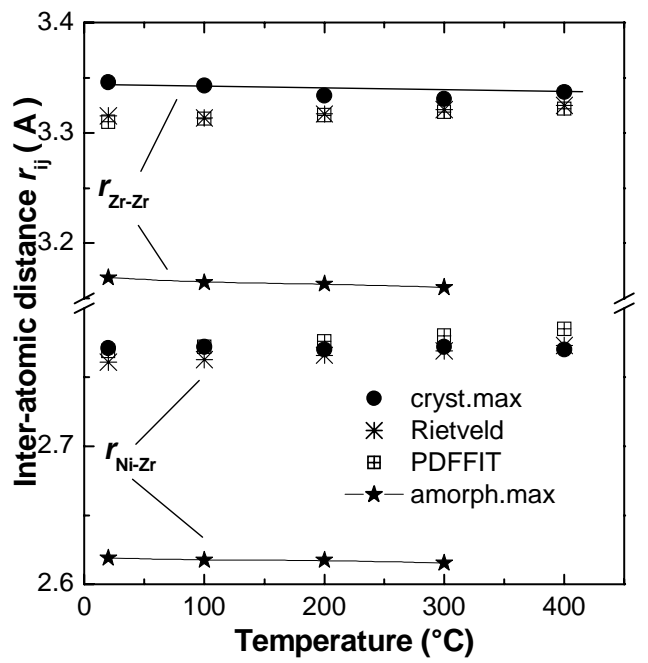

Figure 6. Interatomic distances of $\mathrm{Ni}_{33} \mathrm{Zr}_{67}$.

The atomic pair correlation functions $G(r)$ of the crystallized material were analyzed by a fit of the crystal structure parameter using PDFFIT [7]. Figure 5 shows one of the resulting plots. From the results of the real space fit procedure the inter-atomic distances can be calculated. Table 2 and figure 6 compare the values of the nearest neighborhood calculated by different methods. The positions of the two components of the first maximum of experimental $G(r)$ decrease slightly with increasing temperature (* amorphous, $\bullet$ crystalline). From the crystal structure of $\mathrm{NiZr}_{2}$ follows that the first coordination sphere consists of 6 different

Table 1. Interatomic distances in of amorphous and crystallized $\mathrm{Ni}_{33} \mathrm{Zr}_{67}$

\begin{tabular}{|c|c|c|c|c|c|c|}
\hline & $\left(\mathrm{N}_{\mathrm{ij}}\right)$ & rt. & $100^{\circ} \mathrm{C}$ & $200^{\circ} \mathrm{C}$ & $300^{\circ} \mathrm{C}$ & $400^{\circ} \mathrm{C}$ \\
\hline \multirow[t]{2}{*}{ amorphous } & $r_{\mathrm{Ni}-\mathrm{Zr}}^{1} / \AA$ & 2.619 & 2.618 & 2.617 & 2.615 & - \\
\hline & $r_{\mathrm{Zr}-\mathrm{Zr}}^{1} / \AA \quad(11)$ & 3.169 & 3.164 & 3.163 & 3.160 & - \\
\hline \multirow[t]{2}{*}{ crystalline } & $r_{\mathrm{Ni}-\mathrm{Zr}}^{1} / \AA$ & 2.771 & 2.772 & 2.770 & 2.772 & 2.770 \\
\hline & $r_{\mathrm{Zr}-\mathrm{Zr}}^{1} / \AA(11)$ & 3.346 & 3.343 & 3.334 & 3.321 & 3.337 \\
\hline \multirow{6}{*}{$\begin{array}{l}\text { crystalline } \\
\text { Rietveld }\end{array}$} & $r_{\mathrm{Ni}-\mathrm{Zr}}^{1} / \AA$ & 2.761 & 2.763 & 2.766 & 2.769 & 2.772 \\
\hline & $r_{\mathrm{Zr}-\mathrm{Zr}}^{1} / \AA$ & 3.011 & 3.013 & 3.016 & 3.023 & 3.024 \\
\hline & (2) & 3.071 & 3.073 & 3.076 & 3.079 & 3.082 \\
\hline & (4) & 3.387 & 3.389 & 3.392 & 3.396 & 3.400 \\
\hline & (4) & 3.432 & 3.434 & 3.438 & 3.443 & 3.447 \\
\hline & $r_{\mathrm{Ni}-\mathrm{Ni}}^{1} / \AA$ & 2.639 & 2.640 & 2.641 & 2.642 & 2.643 \\
\hline \multirow{6}{*}{$\begin{array}{l}\text { crystalline } \\
\text { PDFFIT }\end{array}$} & $r_{\mathrm{Ni}-\mathrm{Zr}}^{1} / \AA$ & 2.769 & 2.772 & 2.776 & 2.780 & 2.785 \\
\hline & $r_{\mathrm{Zr}-\mathrm{Zr}}^{1} / \AA$ & 2.958 & 2.962 & 2.951 & 2.947 & 2.941 \\
\hline & (2) & 3.104 & 3.105 & 3.117 & 3.124 & 3.133 \\
\hline & (4) & 3.370 & 3.372 & 3.372 & 3.372 & 3.373 \\
\hline & (4) & 3.442 & 3.446 & 3.450 & 3.457 & 3.462 \\
\hline & $r_{\mathrm{Ni}-\mathrm{Ni}}^{1} / \AA$ & 2.642 & 2.643 & 2.649 & 2.651 & 2.655 \\
\hline
\end{tabular}


inter-atomic distances: $\mathrm{Ni}-\mathrm{Ni}(\mathrm{N}=2$ at $2.64 \AA$ ̊), $\mathrm{Ni}-\mathrm{Zr}(\mathrm{N}=8$ at $2.77 \AA), \mathrm{Zr}-\mathrm{Zr}(\mathrm{N}=1$ at $3.01 \AA), \mathrm{Zr}-\mathrm{Zr}(\mathrm{N}=2$ at $3.10 \AA), \mathrm{Zr}-\mathrm{Zr}(\mathrm{N}=4$ at $3.39 \AA)$, and $\mathrm{Zr}-\mathrm{Zr}(\mathrm{N}=4$ at $3.43 \AA)$. These individual distances are not resolved in the experimental $G(r)$ curve. The inter-atomic distances can be calculated from the results of the pair correlation function fit as well as from the results of the Rietveld refinement (table 2). There is a general good agreement between both methods within about $\pm 0.01 \AA$. Reciprocal and real space data analysis shows an increase of the partial inter-atomic distances with temperature. The weighted averages (by coordination number $\mathrm{N}$ ) of the $\mathrm{Zr}-\mathrm{Zr}$ distances are shown in figure 6 ( : Rietveld, $\exists$ : PDFFIT). The comparison with the behavior of the first maximum positions of the measured atomic pair correlation function $G(r)$ shows differences especially for the temperature dependence. Obviously the values of the maxima position of the experimental $G(r)$ curve give only a rough estimate of the inter-atomic distances. The total pair correlation functions have a complicated shape from the superimposition of different distances unable to fit with simple mathematic functions. With increasing temperature the width of the distance distribution increases due to the enhanced thermal oscillations of the atoms that affects the maxima position additionally.

\section{Conclusions}

The short-range order in amorphous and in crystallized $\mathrm{Ni}_{33} \mathrm{Zr}_{67}$ is quite different. The analysis of the atomic pair correlation function of the crystallized state in real space agrees well with those of the Rietveld refinement in reciprocal space. The atomic pair correlation function $G(r)$ contains contributions of different distances, which cannot be resolved in the experimental data. In the case of complex structures, the maxima positions of $G(r)$ and their temperature dependences are not directly related to the behavior of the individual interatomic distances.

\section{References}

1. Mattern, N., Eckert, J., Kühn, U., Hermann, H., Sakowski, J., Herms, G. \& Neuefeind, J., 2002, Appl. Phys. Lett. 80, 4525.

2. Schenk, T., Holland-Moritz, T.D., Simonet, V., Bellissent, R. \& D.M. Herlach, D.M., 2002 Phys. Rev. Lett. 89. 075507.

3. Inoue, A. \& Takeuchi, A., 2002, Mater. Trans. JIM 43, 1892.

4. Sakowski, J., \& Herms, G., 2000, in: "Hasylab Annual Report", Part 1, 985.

5. Wagner, C.N.J., 1972, in "Liquid Metals, Chemistry and Physics", edited by S.Z. Beer (Marcel Dekker Inc., New York), 257.

6. Billinge, S.J.L., 1998, in „Local Structure from Diffraction“, edited by S.J.L. Billinge \& M.F. Thorpe ( New York, Plenum ) 137.

7. Proffen, T., Billinge, S.J.L., 1999, J. Appl.Cryst.32 , 572.

Acknowledgements. The author N.M. is grateful to R.B. Neder and T. Proffen for supplying us with the PDFFIT program and for valuable discussions. 